\title{
Economia política da disputa por terras em Minas Gerais ${ }^{1}$
}

\author{
Ari Francisco de Araujo Junior ${ }^{2}$ \\ Claudio Shikida ${ }^{3}$ \\ Patrícia Silva Alvarenga ${ }^{4}$
}

Resumo: O artigo estuda os determinantes da probabilidade de ocorrência de conflitos de terra, ocupações e assentamentos nos municípios mineiros. Os resultados apontam para a importância de fatores econômicos e políticos. Aparentemente, militantes pró-reforma agrária se adequam ao ciclo político, causando menos conflito em municípios governados por aliados. Por sua vez, a pobreza e o elevado crescimento arrefecem o ímpeto desses militantes.

Palavras-chave: desenvolvimento agrário, economia política, economia regional

Classificação JEL: D72, D74, O43

\footnotetext{
Abstract: The article analyzes the determinants of the probability of

${ }^{1}$ Os autores agradecem os comentários de Fábio Augusto Reis Gomes, Roberta Vieira Raggi e a assistência de pesquisa de Cristiane Mendonça Carneiro. Agradecem também aos participantes da XI Conferência Anual da ALACDE pelos comentários a uma versão anterior deste artigo.

${ }^{2}$ Endereço para Correspondência: Ibmec Minas, Rua Paraíba, 330 - 4 andar Edifício Seculus Business Center, CEP: 30130-140, Belo Horizonte (MG). E-mail: arifaj@ibmecmg.br

${ }^{3}$ Ibmec Minas

${ }^{4}$ UNI-BH
} 
dispute over land (conflicts, occupations and settlement projects) in the Brazilian state of Minas Gerais. Through the use of a logit model, we found that the main influences are political and economical ones. Apparently, the behavior of the agrarian reform's supporters follows the political incentive, with fewer occurrences of conflicts in towns governed by political allies. By other hand, the economical determinants - degree of poverty and the economic growth - have negative impacts on it.

Key words: agrarian development, political economy, regional economics

\section{Introdução}

“...in considering possible income redistribution programs, account must be taken not only of the political problems and the work incentive effects but also the potential use of force as individuals readjust their wealth positions".

[UMBECK, J. (1981), 57]

Toda sociedade apresenta problemas econômicos que envolvem perguntas clássicas sobre "o que, como e para quem produzir". Tradicionalmente, essas questões fazem parte dos estudos sobre o funcionamento dos mercados e dos governos. Em situações nas quais o mercado é incapaz de alocar recursos eficientemente (falhas de mercado), fala-se da necessidade da intervenção governamental, embora esta também possa apresentar problemas (falhas de governo).

Ambas as análises partem do pressuposto de que os membros da sociedade aceitam pacificamente alterar a alocação de direitos de propriedade. Na aquisição de um imóvel, por exemplo, o comprador adquire do vendedor o direito à propriedade do bem e, se eles respeitam a lei, aceitam também que a transação tenha parte de seu valor redirecionada ao governo através do Imposto de Transmissão de Bens Inter Vivos.

Entretanto, existe uma terceira forma de se alocar recursos além das tradicionais trocas voluntárias (mercado) e involuntárias, mas consentidas (governo/sistema político): a troca involuntária e não-consentida, que se refere às alocações realizadas sem o respeito aos direitos de propriedade previamente estabelecidos. A invasão de um terreno, sem autorização de seu dono (ou das autoridades legais) e o assalto à mão 
armada são dois exemplos deste tipo de alocação, independentemente das motivações desses atos.

Observa-se também que essa forma de alocação - que pode ser chamada de economia do conflito - nem sempre é independente do sistema político. É possível que determinados grupos de interesse apóiem a intensificação de conflitos em torno de (re)alocações dos direitos de propriedade com fins políticos.

Neste artigo, busca-se analisar os determinantes de uma das modalidades mais freqüentes de alocação feitas, ao menos inicialmente, a despeito da lei: as invasões de terras promovidas pelo Movimento dos Trabalhadores Rurais Sem-Terra (MST) em Minas Gerais.

Para alguns defensores da reforma agrária, a mesma seria um objetivo urgente de política pública, o que amenizaria, por assim dizer, os desrespeitos infligidos aos direitos de propriedade sobre a terra ${ }^{5}$. Para outros, a reforma agrária é guiada por objetivos ideológicos subjacentes $^{6}$. Este artigo parte de um ponto de vista distinto, considerando que custos e benefícios, tanto políticos como econômicos, guiam as ações do MST, o que as tornam objeto de análise típico da Escolha Pública?

A próxima seção faz uma revisão da literatura econômica sobre o tema. Em seguida, descreve-se a base de dados utilizada, estima-se o modelo e analisam-se os resultados. Finalmente, a última seção apresenta as conclusões.

\section{Revisão da literatura}

Os estudos sobre os determinantes do conflito rural no Brasil sob a ótica econômica da Nova Economia Política são recentes e devemse, basicamente, aos trabalhos de Alston, Libecap \& Schneider (1995)

\footnotetext{
${ }^{5}$ Em termos econômicos, tratam-se de análises carregadas de conteúdo normativo. Ver: http://www.cptnac.com.br/?system $=$ news\&eid $=25$.

${ }^{6}$ Rodriguez (2006).

7 Também conhecida como Economia Política, Nova Economia Política ou Economia Política Positiva. A literatura econômica cada vez mais reconhece os termos como sinônimos. Uma conceituação detalhada do termo encontra-se em Drazen (2000). Vale ressaltar que não é objetivo deste trabalho fazer uma ampla resenha sobre a evolução da economia política, desde Adam Smith até os dias atuais, ou de seus reflexos na questão agrária brasileira. Para um resumo desta última, no âmbito da "antiga/tradicional" Economia Política brasileira, ver Bielschowsky (1988).
} 
e Alston, Libecap \& Mueller (1997, 1999, 2005). Desse conjunto de estudos, aqueles que se encontram mais diretamente relacionados ao presente trabalho são Alston, Libecap \& Mueller $(1999,2005)^{8}$.

Alston, Libecap \& Mueller (1999) ${ }^{9}$ apresentam um modelo microeconômico, no qual donos de terras ("fazendeiros") e invasores otimizam o uso da violência, dados certos parâmetros institucionais. Entre os diversos resultados, cabe destacar:

(...) a change that makes the courts more responsive to land reform and squatter's rights, will not only increase the amount of effort from the squatters, but may also lead to more effort from the farmers, leading to an overall increase in violent conflicts (Alston, Libecap \& Mueller, 1999, 149).

Desse modo, por mais bem-intencionada que seja, a atuação governamental pode ter conseqüências indesejáveis e estas, por sua vez, podem ser atribuídas à (má) formatação dos incentivos que a reforma agrária, tal como tem sido feita, representa para os atores no meio rural $^{10}$.

É interessante notar que, embora o discurso dos invasores de terra seja radicalmente anticapitalista, há evidências na literatura de que a posse da terra altera as preferências dos invasores em direção ao respeito pela importância dos direitos de propriedade privados [Di Tella, Galiani \& Schargrodsky (2004)] ${ }^{11}$. Isso reforça o argumento de Alston,

\footnotetext{
${ }^{8}$ Alston, Libecap \& Mueller (1997) não abordam, especificamente, o problema da reforma agrária relacionado ao MST.

${ }^{9}$ No Anexo 1, é apresentado, de forma resumida, o modelo de Alston, Libecap \& Mueller (1999).

${ }^{10}$ Efeitos adversos da ação governamental sob os mercados são bastante conhecidos e documentados na literatura. Entretanto, o efeito adverso de ações governamentais em situações nas quais o direito de propriedade está em disputa de forma violenta pode-se apresentar como um resultado ligeiramente distinto e interessante. Kirk (1983) mostrou que o aumento do tamanho do governo, ao invés de coibir, pode estimular ações terroristas.

${ }^{11}$ Os efeitos da obtenção dos direitos de propriedade sobre o desempenho socioeconômico dos antigos invasores dependem da amostra em questão. Galiani \& Schargrodsky (2005) é um exemplo deste tipo de análise para a Argentina, estendendo o estudo de Di Tella, Galiani \& Schargrodksy (2004). Para Minas Gerais, Silva, Coelho \& Alves Filho
} 
Libecap \& Mueller (1999): a busca por terra pode ser pensada como uma disputa por direitos de propriedade.

Outro aspecto característico do modelo dos autores é que a disputa pelos direitos de propriedade não se dá sobre um bem de uso comum, como geralmente aparece na literatura da Economia do Conflito [Umbeck (1981), Hirshleifer (2001), Skaperdas (2003)]. Trata-se do uso de violência para alterar a estrutura de direitos de propriedade já existente, com o uso alternativo da influência política. A análise econométrica utilizou dados estaduais (22 estados) no período de 1988 a 1995. Os principais resultados do artigo foram: (i) aumentos no orçamento do Incra (uma proxy da ação governamental pró-reforma agrária) geram aumentos no número de conflitos; (ii) aumentos do preço da terra levam a maiores conflitos de terras, (iii) maior proteção aos direitos de propriedade gera menos conflitos e (iv) um número maior de fazendas em um estado incentiva a ocorrência de conflitos.

Em Alston, Libecap \& Mueller (2005), os autores abordam outro aspecto do problema dos conflitos de terra, estudando o paradoxal sucesso do MST, já que se trata de um grupo que não recebe votos ou, aparentemente, fundos que poderiam ser usados para eleger políticos que lhe são favoráveis ${ }^{12}$. Nas palavras dos autores:

The MST is a well-organized interest group with a comparative advantage in influencing the information received by voters. This is done through highly-publicized farm occupations, marches, invasions of governmental offices, roadblocks, and accusations that the government is stalling. Through these actions voters revised their views regarding the government's commitment toward land reform and increased their

(2006) analisaram o caso de uma cooperativa agroindustrial da cidade de João Pinheiro (“projeto Furta D’Anta”) e concluíram que a mesma teria sido bem-sucedida na sua sustentabilidade por apresentar uma administração de características empreendedoras.

${ }^{12}$ Em resumo, a parcela urbana do eleitorado apresenta alto custo de oportunidade de se informar sobre a situação de conflitos no campo, o que, em conjunto com uma ação racional do movimento sobre a mídia, resulta em ganhos para o movimento, mesmo que este não seja legalmente representado por nenhum partido político no Congresso Nacional. Nesse sentido, seu artigo apresenta certo parentesco com o já citado Kirk (1983), que modelou a disputa entre um governo e um grupo de interesse que poderia optar entre o uso da violência (“terrorismo”) ou a ação política (lobby, partidos políticos). 
political pressure for more action. In this new scenario the equilibrium level of government effort for the land reform 'task' is greater than in the pre-MST scenario (Alston, Libecap, \& Mueller, 2005, 16).

Neste artigo, a ação do governo é tornada endógena através de um modelo de múltiplos agentes e múltiplos principais. A influência do MST na mídia é destacada pelos autores e encontra-se resumida no Quadro 1.

Quadro 1 - MST e a Mídia

\begin{tabular}{|c|c|}
\hline Características & Resumo \\
\hline $\begin{array}{l}\text { Baixo custo marginal de } \\
\text { influenciar a informação. }\end{array}$ & $\begin{array}{l}\text { - Independente da concordância ou não dos } \\
\text { eleitores, o MST possui a tecnologia de obter } \\
\text { extensa cobertura da mídia para seus atos. } \\
\text { Um dos motivos é o fato de as invasões e } \\
\text { ocupações serem bastante trabalho-intensiva, } \\
\text { o que aumenta a visibilidade das mesmas }{ }^{13} \text {. } \\
\text { Outro é o baixo custo de oportunidade dos } \\
\text { camponeses sem-terra ("exército camponês } \\
\text { de reserva") relativamente ao custo dos } \\
\text { fazendeiros. }\end{array}$ \\
\hline $\begin{array}{l}\text { Produtividade do esforço } \\
\text { empregado para influenciar a } \\
\text { informação. }\end{array}$ & $\begin{array}{l}\text { - Na prática, o MST consegue mais visibilidade } \\
\text { favorável que os fazendeiros. Um exemplo } \\
\text { foi o papel positivo divulgado em novela } \\
\text { de grande rede de comunicação nacional. } \\
\text { Fazendeiros não têm conseguido o mesmo } \\
\text { resultado, tendo se concentrado na arena } \\
\text { política. }\end{array}$ \\
\hline $\begin{array}{l}\text { Extrema configuração das } \\
\text { preferências dos eleitores. }\end{array}$ & $\begin{array}{l}\text { - Eleitores são favoráveis à reforma agrária } \\
\text { e esta é, empiricamente, uma variável que } \\
\text { aumenta a popularidade presidencial. }\end{array}$ \\
\hline
\end{tabular}

Fonte: Elaboração dos autores com base em Alston, Libecap \& Mueller (2005).

Finalmente, a análise econométrica de Alston, Libecap \& Mueller (2005) não refutou as evidências de que ocupações influenciam a popularidade presidencial de maneira significativa:

13 "O mais importante, contudo, é que cada passo sempre leva em conta o apelo jornalístico, pois os estrategistas do MST sabem muito bem que um acampamento à beira de uma rodovia tem muito mais visibilidade do que no interior das terras, do mesmo modo que montar um acampamento provisório em frente ao palácio do governo, ou diante de órgãos do governo ou agências bancárias, garante presença no noticiário da televisão e nos jornais" (Comparato, 2001, 111). 
The estimation indicates that a one standard deviation increase of the number of farms occupied, with all other variables at their means, reduces presidential popularity by 6.22 percentage points from $60.9 \%$ to $54.7 \%$. This is a strong effect for a non-economic variable that doesn't really affect most (urban) voters' well-being directly (Alston, Libecap \& Mueller, 2005, 27).

Sucintamente, a literatura resenhada mostra que o correto delineamento dos direitos de propriedade sobre um recurso como a terra apresenta conseqüências importantes. Compreender a economia política - tal como entendida neste $\operatorname{artigo}^{14}$-, que determina que a oferta e a demanda de políticas públicas neste setor ${ }^{15}$ são essenciais não apenas para a compreensão do tema, mas também para a proposição de políticas públicas ${ }^{16}$.

Nesse sentido, é interessante que estudos similares sejam realizados para cortes geográficos diferentes, algo que não foi feito pelos autores resenhados. Assim, uma das contribuições deste artigo é retomar Alston, Libecap \& Mueller (1999), mas no contexto estadual, a partir de uma base de dados municipal e focada no caso de Minas Gerais.

A seção seguinte detalha melhor esta base e a metodologia econométrica adotada.

\section{A econometria da disputa por terras em Minas Gerais ${ }^{17}$}

\subsection{Definições das variáveis e suas fontes}

A base de dados foi construída através de uma cross-section dos 853 municípios mineiros no ano de 2000. As variáveis dependentes binárias

\footnotetext{
${ }^{14}$ Ver nota de rodapé 7 .

${ }^{15}$ Os problemas da violência no campo e da reforma agrária, obviamente, apresentam outras dimensões importantes de análise que são, temporalmente, posteriores ao foco deste artigo. Como exemplos, cita-se a importância do "capital social” [Barreto, Khan \& Lima (2005)] e da "urbanização" do meio rural [(Souza \& Del Grossi (2002)].

${ }^{16}$ Em termos históricos, a disputa pelos direitos de propriedade da terra é antiga no Brasil e provavelmente sofre de path dependence, ou seja, é fortemente influenciada por instituições do passado colonial. Mueller (2005) e Rezende (2006) ilustram bem a importância de antigas instituições na formatação dos incentivos atuais que estimulam o conflito no campo.

${ }^{17}$ Uma análise positiva (no sentido econômico) da situação da violência no campo no Brasil apresenta uma limitação importante: a qualidade das bases de dados.
} 
(conf, ocup e assent) têm como fonte primária o Incra ${ }^{18}$ (Instituto Nacional de Colonização e Reforma Agrária) e a $\mathrm{CPT}^{19}$ (Comissão Pastoral da Terra). Definem-se da seguinte forma:

conf $=1$, caso tenha sido observado pelo menos um conflito de terras em determinado município no ano de 2000 e, conf $=0$, caso contrário ${ }^{20}$; ocup $=1$, caso tenha sido observada pelo menos uma ocupação de terras em determinado município no ano de $2000^{21} \mathrm{e}$, ocup $=0$, caso contrário.

assent00 $=1$, caso tenha sido estabelecido pelo menos um assentamento em determinado município no ano de $2000^{22}$ e, assent00 $=0$, caso contrário.

O objetivo é estimar os determinantes da probabilidade de ocorrência de ocupações, conflitos de terra e assentamentos em Minas Gerais. Para o caso específico dos assentamentos, talvez seja razoável supor que as condições econômicas e políticas observadas em 2000 surtam efeito em termos do estabelecimento de assentamentos com alguma defasagem de tempo. Portanto, utilizou-se também a variável de "assentamentos” estabelecidos em 2004 (assent04), ao final dos mandatos iniciados em $2000^{23}$.

\footnotetext{
${ }^{18}$ Ver Incra (http://www.incra.gov.br).

${ }^{19}$ Ver CPT (http://www.cptnac.com.br/?system = news\&action = read\&id =1266\&eid =6)

${ }^{20} \mathrm{O}$ conceito de conflitos refere-se ao que a CPT chama de "conflitos por terra": "São ações de resistência e enfrentamento pela posse, uso e propriedade da terra e pelo acesso a seringais babaçuais ou castanhais, quando envolvem posseiros, remanescentes de quilombos, parceleiros, assentados, pequenos arrendatários, pequenos proprietários, sem-terra, ocupantes, seringueiros, quebradeiras de coco babaçu, castanheiros, índios, entre outros". (http://www.cptnac.com.br/?system $=$ news\&action $=$ read\&id $=1262 \&$ ei $\underline{\mathrm{d}=6}$. .

${ }^{21}$ Conforme a CPT, define-se ocupações como: “Ações coletivas de famílias sem-terra que, por meio da entrada em imóveis rurais, reivindicam terras que não cumprem a função social". (http://www.cptnac.com.br/?system $=$ news\&action $=$ read\&id $=1266 \& \mathrm{e}$ $\underline{\mathrm{id}=6}$ )

${ }^{22}$ Considera-se assentamento áreas em que houve desapropriação da terra e criação do PA - Projeto de Assentamento pelo Incra. Essas áreas foram inseridas oficialmente em programas de reforma agrária do governo.

${ }^{23}$ Uma variável não incluída na regressão, mas que pode ser interessante para futuros trabalhos é o total de recursos federais recebidos pelo MST. Segundo noticiou a imprensa [Gripp (2006)], o aumento de verbas foi incapaz de diminuir as invasões no campo.
} 
A Tabela 1 apresenta algumas estatísticas descritivas sobre a disputa de terras em Minas Gerais. Em média, menos de 2\% dos municípios mineiros tiveram algum tipo de conflito por terras, enquanto pouco mais de $4 \%$ deles sofreram ocupação em 2000. Vale notar, portanto, que pode ocorrer conflito sem sucesso quanto à ocupação da terra. $\mathrm{O}$ contrário também pode ser verdadeiro: uma área pode ser ocupada sem que aconteça algum tipo de embate entre os representantes do movimento e os fazendeiros e seus funcionários. Por outro lado, a média de municípios que tiveram o estabelecimento de pelo menos um assentamento foi inferior a $1 \%$ em 2000. Em 2005, passou para $1,4 \%$ em 2005, ou seja, um aumento superior a $40 \%$.

Tabela 1 - Estatísticas Descritivas

\begin{tabular}{llll}
\hline & \multicolumn{3}{c}{ Disputa de Terra em Minas Gerais } \\
\hline Variável & Obs & Média & Des.Pad \\
conf & 853 & .042204 & .1357464 \\
ocup & 853 & .042204 & .2011722 \\
assent00 & 853 & .0093787 & .0964448 \\
assent04 & 853 & .014068 & .1178404 \\
\hline
\end{tabular}

Fonte: Elaboração própria.

A primeira variável explicativa da probabilidade de conflitos, ocupações e assentamentos utilizada foi o Módulo Fiscal (mod). O módulo fiscal constitui uma referência de área estabelecida pelo Incra que varia regionalmente. É definido para cada município, a partir de vários fatores, tais como: qualidade do solo, relevo, condições de acesso ao local, entre outros. Esta variável pode ser entendida como o inverso da produtividade, já que, quanto maior o módulo fiscal, maior a quantidade de terra necessária para sustentar uma família no local. O sinal esperado, neste caso, é ambíguo, tendo em vista que pode-se pensar que os conflitos e ocupações têm maior chance de ocorrer em terras melhores, mais produtivas (portanto, sinal negativo) ou, do ponto de vista mais político, em terras de pior qualidade (portanto, sinal positivo), nas quais o proprietário não demonstra muita resistência para defendê-

Pode-se pensar que se está estimando apenas o efeito-substituição da violência no campo, deixando de lado o efeito-renda. 
la. Ou seja, os custos podem superar os benefícios e, dessa forma, o movimento ganha visibilidade (benefícios políticos), mesmo que não obtenha benefícios econômicos com o assentamento de seus membros em terras desse tipo. No caso dos assentamentos, parece existir uma tendência do Incra adquirir as terras de pior qualidade para a implantação de assentamentos, o que sugere uma relação esperada positiva entre o modulo fiscal e os assentamento ${ }^{24}$. Pode-se notar, na Tabela 2, que o módulo fiscal médio do estado situa-se em torno de 33 hectares. A variável módulo fiscal tem também como fonte o Incra.

Para controlar as diferenças de renda entre os municípios, será utilizado um indicador de pobreza (POB) para o ano de 2000 do Atlas do Desenvolvimento Humano do convênio FJP, Ipea e PNUD. A variável pobreza é definida como a proporção de indivíduos com renda domiciliar per capita inferior a $\mathrm{R} \$ 75,50$ ou a metade do salário mínimo vigente em agosto de 2000. A pobreza municipal média é de pouco mais de $42 \%$ no estado de Minas Gerais (Tabela 2). Como ocorre com outras variáveis, a amplitude (e, conseqüentemente, a desigualdade) da pobreza é bastante elevada. O município com menor proporção de pobres é Poços de Caldas, 8,92\%, enquanto Monte Formoso apresenta proporção de pobres quase 10 vezes superior $(85,02 \%)$.

Além de controlar pelo nível de renda, é importante verificar se o crescimento municipal exerce o mesmo efeito sobre as chances de conflitos, ocupações de terra e assentamento. Para tanto, será utilizada a taxa de crescimento média entre 1991 e 2000 dos municípios mineiros. A fonte desta variável também é o Atlas do Desenvolvimento Humano do convênio FJP, Ipea e PNUD. Pela Tabela 2, nota-se que os municípios mineiros cresceram, em média, 1,25\% ao ano entre 1991 e 2000.

Além dos determinantes socioeconômicos, criou-se uma variável para testar o determinante político dos conflitos, ocupações de terra e assentamentos estabelecidos. Trata-se de uma variável dummy que assume o valor 1 caso o município seja governado em 2000 pelo Partido dos Trabalhadores (PT) e 0 nos casos restantes (d_pt). O objetivo é testar a possível ligação existente entre os movimentos de trabalhadores semterra e as administrações petistas. Em outras palavras, se são maiores ou

$\overline{{ }^{24} \text { Ver Sauer (2002). }}$. 
menores as chances de um município administrado pelo PT ter algum tipo de disputa por terra ou mesmo um assentamento estabelecido. A fonte de tal variável é o Tribunal Superior Eleitoral ${ }^{25}$ (TSE). Também na Tabela 2, é possível observar que pouco menos de $10 \%$ dos municípios mineiros eram administrados pelo PT.

Finalmente, foi criada uma variável que interage o módulo fiscal e a dummy do PT (mod_pt). O objetivo, neste caso, é verificar se o efeito observado para a variável de módulo fiscal se mantém para os municípios administrados pelo PT.

Tabela 2 - Estatísticas Descritivas

\begin{tabular}{llcl}
\hline & \multicolumn{3}{c}{ Variáveis Explicativas } \\
\hline Variável & Obs & Média & Des.Pad. \\
mod & 853 & 33.02345 & 13.7956 \\
pob & 853 & 42.04999 & 18.26037 \\
cresc_m & 853 & 1.240508 & .6078149 \\
d_pt & 853 & .0996483 & .2997062 \\
\hline
\end{tabular}

Fonte: Elaboração Própria.

Resumindo, serão apresentadas as estimativas da seguinte equação:

conf ou ocup ou assent $=$ F (mod, idhe, cresc_m, d_pt, mod_pt $)+\varepsilon$

\subsection{Metodologia}

Com o objetivo de estimar os determinantes da probabilidade de conflito, ocupações de terra e assentamentos em Minas Gerais, analisa-se a correlação, em termos probabilísticos, de variáveis socioeconômicas e políticas com uma variável dependente binária construída para capturar a ocorrência de conflitos, ocupações e assentamentos nos municípios mineiros no ano de $2000^{26}$. Ou seja, trabalha-se com três regressandos distintos.

\footnotetext{
${ }^{25}$ Ver TSE (http://www.tse.gov.br).

${ }^{26}$ No caso de assentamentos, será analisado o problema também supondo a existência de defasagens temporais.
} 
Dessa forma, visando verificar como tais probabilidades são influenciadas entre as unidades de análise, supõe-se a seguinte relação linear:

$$
\mathrm{Y}=\alpha+\beta \mathrm{X}+\xi
$$

em que $\alpha$ e $\beta$ são os respectivos parâmetros a serem estimados, $\mathrm{X}$ é a matriz dos regressores, $\xi$ é a perturbação aleatória e Y é variável dependente que, especificamente, pode ser assim caracterizada:

$\mathrm{Y}=1$, caso tenha ocorrido pelo menos um conflito, ocupação ou assentamento em determinado município mineiro e,

$\mathrm{Y}=0$, caso contrário.

Pode-se, então, expressar as probabilidades de ocorrência/nãoocorrência através das seguintes expressões:

$$
\begin{aligned}
& \operatorname{Pr}(\mathrm{Y}=1)=F(v X) \\
& \operatorname{Pr}(\mathrm{Y}=0)=1-F(v X)
\end{aligned}
$$

De modo que são estimadas:

$$
\mathrm{Y}=F(v X)+\varepsilon
$$

em que $F(v X)$ é a função de distribuição cumulativa de probabilidade. O problema é a escolha da relação funcional apropriada para as distribuições de probabilidade acima que apresente predições consistentes. As formas funcionais mais comuns são a Linear, a Logit a Probit ${ }^{27}$.

O principal problema com a primeira especificação é que ela pode gerar probabilidades estimadas fora do intervalo 0-1, não obedecendo as seguintes características estatísticas esperadas:

$\lim \operatorname{Pr}(\mathrm{Y}=1)=1$

$$
\mathrm{vX} \rightarrow+\infty
$$

${ }^{27}$ As formas funcionais descritas acima são as seguintes: 


$$
\begin{aligned}
& \lim _{\mathrm{vX} \rightarrow-\infty} \operatorname{Pr}(\mathrm{Y}=0)=0
\end{aligned}
$$

Neste exercício, optou-se por estimar o modelo teórico através do Probit por Máxima Verossimilhança - MV. Faz-se também uma análise dos efeitos marginais obtidos a partir do modelo ${ }^{28}$. Como descrito na seção sobre as variáveis utilizadas, a regressão proposta inclui uma variável que interage o módulo fiscal e a dummy de administração petista. Como observado por Ai \& Norton (2003), apesar do largo uso dos termos de interação em trabalhos de econometristas aplicados, a maioria dos pesquisadores não interpreta de modo correto o coeficiente estimado para estes termos em modelos não-lineares. Os autores revisaram 72 artigos publicados em journals de economia listados na base JSTOR e descobriram que em nenhum desses trabalhos o termo de interação foi interpretado corretamente. Os pesquisadores nacionais parecem cometer o mesmo tipo de erro. Em outros casos, a variável interativa é incluída para melhor especificar o modelo, mas não se tem a preocupação de interpretá-la ${ }^{29}$.

Em modelos lineares, o valor esperado da variável dependente condicional às variáveis independentes (incluindo o termo de interação) é dado por:

$$
E\left[Y \mid x_{1}, x_{2}, X\right]=\beta_{1} x_{1}+\beta_{2} x_{2}+\beta_{12} x_{1} x_{2}+X \beta
$$

Se $\mathrm{x}_{1}$ e $\mathrm{x}_{2}$ são variáveis contínuas, o efeito de interação (supondo que $\mathrm{X}$ é independente de $\mathrm{x}_{1}$ e $\mathrm{x}_{2}$ ) das variáveis $\mathrm{x}_{1}$ e $\mathrm{x}_{2}$ é uma derivada cruzada do valor esperado de $\mathrm{Y}$ :

$28 \quad$ Neste caso, o efeito marginal é dado pela seguinte expressão:

$$
\frac{\partial E(Y \mid X)}{\partial x}=\phi(X, \beta) \beta
$$

em que $X \beta$ representa o vetor de coeficientes multiplicado por um vetor que contenha valores para as variáveis dependentes. O efeito marginal (no programa Stata trata-se do comando dprobit) pode ser interpretado como a mudança na probabilidade para uma mudança infinitesimal em cada variável independente para as variáveis contínuas e a mudança discreta na probabilidade para variáveis dummies.

${ }^{29}$ Para o caso de artigos nacionais, ver Nakane, Alencar \& Kanczuk (2005), Botelho \& Urdan (2005), Corseuil, Santos \& Foguel (2001). 


$$
\frac{\partial E\left[Y \mid x_{1}, x_{2}, X\right]}{\partial x_{1} \partial x_{2}}=\beta_{12}
$$

Para o caso em que a interação é feita com variáveis dicotômicas, têm-se diferenças discretas:

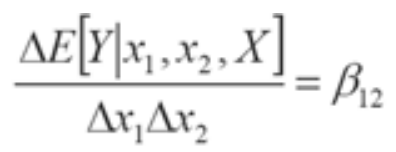

Portanto, um teste t do coeficiente $\beta_{12}$ é suficiente para diagnosticar a significância estatística do efeito da variável de interação. Já em modelos não-lineares, o efeito deve ser calculado de outro modo, tal como proposto em Ai \& Norton (2003). Supondo um modelo Probit (neste caso Y é uma dummy), tem-se:

$$
\begin{aligned}
& E\left[Y \mid x_{1}, x_{2}, X\right]=F\left(\beta_{1} x_{1}+\beta_{2} x_{2}+\beta_{12} x_{1} x_{2}+X \beta\right) \\
& E\left[Y \mid x_{1}, x_{2}, X\right]=F(u)
\end{aligned}
$$

Em que $F$ é a distribuição cumulativa da normal padrão e u é $\left(\beta_{1} x_{1}+\beta_{2} x_{2}+\beta_{12} x_{1} x_{2}+X \beta\right)$. Como afirmam os autores, a maioria dos pesquisadores usa o $\frac{\partial F(u)}{\partial\left(x_{1} x_{2}\right)}=\beta_{12} F^{\prime}(u)$ como efeito de interação. Na

verdade, o efeito completo de interação é a derivada parcial cruzada do valor esperado de $\mathrm{Y}$ :

$$
\frac{\partial F(u)}{\partial x_{1} \partial x_{2}}=\beta_{12} F^{\prime}(u)+\left(\beta_{1}+\beta_{2} x_{2}\right)\left(\beta_{2}+\beta_{12} x_{1}\right) F^{\prime \prime}(u)
$$

Vale notar que o efeito de interação pode ser diferente de zero, mesmo que $\beta_{12}$ seja igual a zero. A significância estatística do efeito da interação deve ser calculada, já que não pode ser testada por um simples teste t do coeficiente do termo de interação $\beta_{12}$. Além disso, o efeito de interação é condicional às variáveis independentes (o que não ocorria no caso de modelos lineares) e pode ter sinais diferentes para distintos valores das variáveis explicativas. Ou seja, o sinal de $\beta_{12}$ não indica necessariamente o sinal do efeito de interação. 
Resumindo, as estimativas dos determinantes da disputa por terras em Minas Gerais serão feitas via modelo Probit (estimado por Máxima Verossimilhança) com correção para heterocedasticidade pela matriz de variância-covariância de White. Adicionalmente, utilizam-se o método e o programa para Stata proposto por Ai \& Norton (2003) e Norton, Wang \& Ai (2004) para interpretar corretamente o termo de interação.

\section{Resultados ${ }^{30}$}

Os resultados das estimativas econométricas das quatro variáveis dependentes são reproduzidos na Tabela 3. Com relação ao conflito de terras, apenas uma variável se mantém significativa: a probabilidade de conflito aumenta com o módulo fiscal (ou seja, a probabilidade aumenta quanto pior a produtividade da terra). Portanto, sob uma ótica mais política, em terras de pior qualidade, o proprietário não demonstra muita resistência e, dessa forma, o movimento age com maior facilidade. Esse resultado é semelhante ao observado para dados estaduais em Alston, Libecap e Mueller (1999). Segundo os autores, terras mais caras e, conseqüentemente, mais desenvolvidas em termos produtivos, são, em média, menos freqüentemente invadidas, já que os movimentos sabem que a probabilidade de sucesso na "empreitada” é menor. Quantitativamente, o aumento de uma unidade no módulo fiscal geraria um aumento de apenas $0,0007 \%$ na probabilidade de ocorrência de conflitos de terra. Vale notar que o pseudo - $\mathrm{R}^{2}$ é de apenas 4,2\%. Também é interessante que, pelos Gráficos 1 e 2 do Anexo 3, apesar de apresentar sinais tanto positivos quanto negativos (na verdade, a maioria é negativo), o efeito de interação não é significativo ao nível de $5 \%$ para nenhum município.

A regressão para ocupações mostra-se bem superior tanto em relação ao pseudo - $\mathrm{R}^{2}(18,1 \%)$ quanto para a significância estatística das variáveis, já que apenas uma delas (mod_pt) não é significativa ao nível de $10 \%$. Neste caso, as chances de ocupação aumentam nas cidades cujas terras apresentam pior qualidade e caem com a pobreza, com o crescimento médio e com a dummy PT. Ou seja, as ocupações são mais freqüentes em municípios ricos, mas estagnados. Além disso,

${ }^{30}$ Correlações bivariadas das variáveis utilizadas encontram-se no Anexo 2. 
os resultados sugerem que os movimentos dos sem-terra "evitam" causar problemas para aqueles municípios cuja administração é petista ${ }^{31}$. Quantitativamente, o aumento de uma unidade no módulo fiscal geraria um aumento de $0,0019 \%$ na probabilidade de ocorrência de ocupações de terra. $\mathrm{O}$ aumento de uma unidade na pobreza geraria uma redução 0,0007\% na probabilidade de ocorrência de ocupações de terra. Já o aumento de uma unidade na taxa de crescimento média reduziria em 0,027\% a probabilidade de ocorrência de ocupações de terra. Finalmente, municípios administrados pelo PT apresentam uma chance 0,027\% menor de ocorrência de ocupações. Em outras palavras, a ordem de magnitude do efeito "político" e a do "crescimento da renda" são os determinantes de maior impacto na probabilidade de ocupação. Apesar de a variável de interação não ser significativa na regressão, pelos Gráficos 3 e 4 do Anexo 3, pode-se notar que o efeito completo de interação é significativo ao nível de $5 \%$ apenas para alguns municípios nos quais o efeito é negativo (ao contrário do sinal do parâmetro estimado no Probit). Ou seja, as chances de ocupação são ligeiramente menores para algumas cidades cujas terras apresentam pior qualidade mas que são administradas pelo PT.

A regressão para assentamentos em 2000 apresenta pseudo - $\mathrm{R}^{2}$ de $21,21 \%$. As variáveis módulo fiscal, pobreza e crescimento médio são significativas estatisticamente. Neste caso, a probabilidade de surgimento de um assentamento em determinado município aumenta com o módulo fiscal e cai com a pobreza e o crescimento médio. Novamente, os assentamentos são mais freqüentes em municípios ricos, mas estagnados. Além disso, as chances são maiores onde as terras são de pior qualidade. Quantitativamente, o aumento de uma unidade no módulo fiscal geraria uma elevação de $0,0003 \%$ na probabilidade de ocorrência de assentamentos. $\mathrm{O}$ aumento de uma unidade na pobreza geraria uma redução de $0,0002 \%$ na probabilidade de ocorrência de assentamentos. Já o aumento de uma unidade na taxa de crescimento média reduziria

\footnotetext{
${ }^{31}$ Podem existir duas interpretações possíveis para o “efeito PT”. Primeiro é possível que prefeituras controladas pelo partido facilitem a invasão e a desapropriação em seus municípios, visando o efeito multiplicador local dos créditos agrícolas. Por outro lado, é possível que o efeito seja oposto na proximidade das eleições. Seria mais interessante estimar o modelo a partir de um painel de dados para se verificar o efeito da proximidade das eleições. Entretanto, isso esbarra nas limitações da base de dados disponíveis.
} 
em $0,005 \%$ a probabilidade de que trabalhadores sem-terra sejam assentados em determinado município. É importante notar que, pelos Gráficos 5 e 6 do Anexo 3, apesar de apresentar sinais positivos, o efeito de interação não é significativo ao nível de $5 \%$ para nenhum município.

Tabela 3 - Regressões Probit com Correção para Heterocedasticidade (White) (estatísticas-z são apresentadas abaixo dos parâmetros estimados)

\begin{tabular}{|c|c|c|c|c|c|c|c|c|}
\hline $\begin{array}{l}\text { Variável } \\
\text { Dependente }\end{array}$ & conf & & ocup & & assent00 & & assent04 & \\
\hline \multirow[t]{2}{*}{$\bmod$} & 0,017915 & $* * *$ & 0,038718 & $*$ & 0,039958 & $*$ & 0,015162 & \\
\hline & 1,890 & & 4,020 & & 3,380 & & 1,200 & \\
\hline \multirow[t]{2}{*}{ pob } & $-0,004768$ & & $-0,014354$ & $* *$ & $-0,019511$ & $* * *$ & $-0,007478$ & \\
\hline & $-0,730$ & & $-2,460$ & & $-1,830$ & & $-0,860$ & \\
\hline \multirow[t]{2}{*}{ cresc_m } & $-0,161015$ & & $-0,552514$ & $*$ & $-0,715492$ & $* *$ & $-0,618261$ & $* *$ \\
\hline & $-0,730$ & & $-3,350$ & & $-2,940$ & & $-2,430$ & \\
\hline \multirow[t]{2}{*}{ d_pt } & 0,830946 & & $-1,341570$ & $* * *$ & $-0,093736$ & & $-0,147600$ & \\
\hline & 1,370 & & $-1,740$ & & $-0,160$ & & $-0,210$ & \\
\hline \multirow[t]{2}{*}{ mod_pt } & $-0,014085$ & & 0,019442 & & 0,009139 & & 0,020023 & \\
\hline & $-0,930$ & & 1,300 & & 0,870 & & 1,190 & \\
\hline \multirow[t]{2}{*}{ constante } & $-2,378947$ & $*$ & $-1,973461$ & $*$ & $-2,472004$ & $*$ & $-1,920626$ & $*$ \\
\hline & $-5,430$ & & $-5,820$ & & $-5,340$ & & $-3,370$ & \\
\hline $\begin{array}{l}\text { No. de } \\
\text { observações }\end{array}$ & 853 & & 853 & & 853 & & 853 & \\
\hline R2 (pseudo) & 0,0424 & & 0,1811 & & 0,2121 & & 0,1417 & \\
\hline
\end{tabular}

FONTE: Elaboração própria a partir dos resultados das regressões.

$(*)$ para p-values menores ou iguais a $\left.0.01 ;{ }^{* *}\right)$ para p-values $<=0.05 ;\left({ }^{* *}\right)$ para p-values $<=0.1$.

Vale enfatizar que pode existir uma defasagem de tempo entre as condições socioeconômicas e políticas e o surgimento de assentamentos. A última regressão da Tabela 3 relaciona as chances de assentamentos serem estabelecidos em 2004 com as condições vigentes em 2000. Neste caso, o pseudo - $\mathrm{R}^{2}$ tem valor em torno dos $14 \%$. Interessante que a única variável (além da constante) que se mantém significativa é o crescimento médio municipal. Uma elevação de uma unidade na taxa de crescimento municipal apresenta chance $0,012 \%$ menor 
de ocorrência de assentamentos. Da mesma forma como observado na regressão, nota-se, pelos Gráficos 7 e 8 do Anexo 3, que o efeito completo de interação não é significativo para nenhum município da amostra.

\section{Conclusão}

A questão agrária é, sem dúvida, um dos maiores problemas socioeconômicos do Brasil. Nesse sentido, é interessante analisar não apenas o desempenho dos assentamentos pós-invasões/assentamentos, mas também os determinantes das ocupações no campo. Este artigo se posiciona neste último tipo de análise: questiona-se sobre os incentivos políticos e econômicos subjacentes aos conflitos sobre os direitos de propriedade de terras no estado de Minas Gerais.

Os resultados apontam para a evidência de que incentivos econômicos e políticos influenciam na decisão de se intensificar ou não o conflito no campo: a pobreza, o crescimento econômico e o suporte político aparecem como significativas estatisticamente e com o sinal esperado na maioria das regressões.

Em outras palavras, a hipótese de que a liderança de MST e similares não levam em conta apenas considerações econômicas para promoverem suas ações, mas agem também com orientações políticas. Dos resultados obtidos, pode-se pensar que políticas voltadas para o crescimento econômico local têm potencial arrefecedor sobre o ritmo do conflito no campo, o que, teoricamente, significa um aumento do custo de oportunidade do uso da violência (trocas involuntárias) relativamente ao uso do mercado (trocas voluntárias) para a obtenção de ganhos de bem-estar.

Finalmente, uma possível extensão deste trabalho consistiria na ampliação da base de dados em termos da dimensão temporal, a fim de verificar a possível existência do ciclo político-econômico sobre as ações dos Sem-Terra. Outro estudo interessante seria utilizar uma base de dados ampliada também em termos municipais para verificar a existência (ou não) de padrões distintos dos conflitos em termos regionais. Vale notar que os resultados não podem ser generalizados para o Brasil devido a diferentes estratégias regionais dos movimentos sem-terra e das políticas públicas locais. 


\section{Referências bibliográficas}

AI, C. \& NORTON, E.C. (2003) Interaction terms in logit and probit models. Economics Letters, v.80, p.123-129.

ALSTON, L., LIBECAP, G. \& MUELLER B. (1999) A Model of Rural Conflict: Violence and Land Reform Policy in Brazil, Environment and Development Economics, Cambridge:UK, 4, p.135-160.

ALSTON, L., LIBECAP, G. \& MUELLER, B. (1997) Violence and the Development of Property Rights to Land in the Brazilian Amazon. In: Nye, J.V.C. and Drobak, J.N., editors, Frontiers of the New Institutional Economics, Academic Press, San Diego, p.145-164.

ALSTON, L.J., LIBECAP, G.D. \& MUELLER, B. (2005) How Interest Groups with Limited Resources can Influence Political Outcomes: Information Control and the Landless Peasant Movement in Brazil. Institute of Behavioral Science, Working Paper 2005-0005.

ALSTON, L.J., LIBECAP, G.D. \& SCHNEIDER, R. (1995) Property Rights and the Preconditions for Markets: The Case of the Amazon Frontier. Journal of Institutional and Theoretical Economics, v.151, n.1, p.89-107.

BARRETO, R.C.S. ; KHAN, A.S. \& LIMA, P.V.P.S. (2005) Sustentabilidade dos assentamentos no município de Caucaia-CE. Revista de Economia e Sociologia Rural, v.43, n.2, p.225-247.

BIELSCHOWSKY, R. (1988) Pensamento Econômico Brasileiro - o ciclo ideológico do desenvolvimentismo. PNPE/IPEA, Rio de Janeiro.

BOTELHO, D., URDAN, A. T. (2005) Lealdade à marca e sensibilidade ao preço: um estudo da escolha da marca pelo consumidor. Revista de Administração Contemporânea, v. 9, n. 4, p. 163-180.

COMPARATO, B.K. (2001) A ação política do MST. São Paulo em Perspectiva, v.15, n.4, p.105-118.

CONNING, J.H. \& ROBINSON, J.A. (2005) Property Rights and the Political Organization of the Agriculture, mimeo. 
CORSEUIL, C.H., SANTOS, D.D. \& FOGUEL, M.N. (2001) Decisões Críticas em Idades Críticas: a Escolha dos Jovens entre Estudo e Trabalho no Brasil e em outros Países da América Latina. Texto Para Discussão n ${ }^{0} 797$, IPEA.

DI TELLA, R., GALIANI, S. \& SCHARGRODSKY, E. (2004) Property Rights and Beliefs: Evidence from the Allocation of Land Titles to Squatters. Ronald Coase Institute. Working Paper \#5.

FETAEMG. Histórico - a construção do movimento sindical. < http:// www.fetaemg.org.br/historico.html > . Acesso em 02.10.2007.

GALIANI, S. \& SCHARGRODSKY, E. (2005) Property Rights for the Poor: Effects of Land Titling. Ronald Coase Institute. Working Paper \#7.

GARFINKEL, M.R. \& SKAPERDAS, S. (ed.) (1996) The political economy of conflict and appropriation. Cambridge University Press.

GRIPP, A. (2006) Mais verbas, mais invasões. O Globo, 18.06.06. Disponível em: http://www.e-agora.org.br/conteudo.php?cont= clipping \&id=4301_0_29_0_M

GROSSMAN, H.I. \& KIM, M. (1996) Predation and production. In: GARFINKEL, M.R. \& SKAPERDAS, S. (ed.) The political economy of conflict and appropriation. Cambridge University Press.

GROSSMAN, H.I. (1994) Production, Appropriation, and Land Reform. American Economic Review, v.84 (3), p.705-712.

HIRHSLEIFER, J. (1991) The paradox of power. Economics and Politics, v.3, p.177-200. [Reproduzido em HIRSHLEIFER, J. The Dark Side of the Force, Cambridge University Press, 2001]

HOROWITZ, A.W. (1993) Time path of land reform: a theoretical model of reform dynamics. American Economic Review, v.83(4), p.1003-1010.

MUELLER, B. (2005) A Evolução Histórica dos Direitos de Propriedade sobre Terras no Brasil e EUA. (mimeo).

MUELLER, B., ALSTON, L., LIBECAP, G. AND SCHNEIDER, R. (1994) "Land Property Rights and Privatization in Brazil". The Quarterly Review of Economics and Finance, v.34, p.261-280. 
MUELLER, D.C. (2003) Public Choice III. Cambridge University Press.

NAKANE, N.I \& ALENCAR, L.S. \& KANCZUK, F. (2005) Demanda por Produtos Bancários e Poder de Mercado na Indústria Bancária Brasileira, (mimeo).

NORTON, E.C, WANG, H. \& AI, C. (2004) Computing interaction effects and standard errors in logit and probit models. Stata Journal, v.4, n.2, p.154-167.

REZENDE, G.C. de. (2006) Pobreza e desigualdade no Brasil: o papel adverso das políticas trabalhista, fundiária e de crédito agrícola. (mimeo).

RODRIGUEZ, R.V. (2006) O governo Lula e os movimentos sociais. Banco de Idéias, ano X, n.36, p.9-11.

SAUER, S. A (2002) Terra por uma Cédula: Estudo sobre a Reforma Agrária de Mercado. (mimeo). Disponível em: http://www.landaction.org/gallery/A \%20TERRA \% 20POR\%20UMA_\%20DE\%20MERCADOPort-Sauer.pdf

SILVA, E.H. de, COELHO, F.M.G. \& ALVES FILHO, E. (2006) Inovação e Sustentabilidade Econômica em Projetos de Assentamento da Reforma Agrária. Revista Econômica do Nordeste, v.37, n.2, p.216-233.

SKAPERDAS, S. (2003) Restraining the Genuine Homo Economicus: Why the Economy Cannot Be Divorced from Its Governance. Economics and Politics, v.15, n.2, p. 135-162.

SOUZA, M. de \& DEL GROSSI, M.E. (2002) A evolução das ocupações das famílias na região não-metropolitana rural do Estado do Paraná: 1992-99. Revista de Economia e Sociologia Rural, v.40, n.4, p.807822.

UMBECK, J. (1981) Might makes rights: a theory of the formation and initial distribution of property rights. Economic Inquiry, v.19, p. 38-59. 
Anexo 1 - Modelo de Alston, Libecap \& Mueller (1999)

Alston, Libecap \& Mueller (1999) apresentam um modelo microeconômico, no qual donos de terras (fazendeiros) e invasores otimizam o uso da violência, dados certos parâmetros institucionais. De forma resumida, o modelo consiste na solução do seguinte par de equações:

$$
\begin{aligned}
& \underset{s}{\operatorname{Max}}(1-\beta(v, K))[(1-\theta(s, P, G)) \pi L+\theta(s, P, G) L]-C^{F}(s) \\
& \operatorname{Max}_{v} \beta(v, K) L+(1-\beta(v, K))[(1-\theta(s, P, G)) \delta L+\theta(s, P, G) \gamma L]-C^{F}(v)
\end{aligned}
$$

sendo que:

$0<\pi<1, \quad 0<\delta<1, \quad \gamma<\delta<1, \quad \theta_{s} \geq 0, \quad \theta_{s s}<0, \quad \theta_{G}>0, \quad \theta_{s G}>0$, $\beta_{v} \geq 0, \quad \beta_{v v}<0, \quad \beta_{K} \leq 0, \beta_{v K} \leq 0, C_{s}^{S}>0, C_{v}^{F}>0, \quad C_{s s}^{S}>0$, $C_{v v}^{F}>0$.

$\mathrm{P}=$ nível de tenure security do pedido do fazendeiro à Justiça

$\mathrm{G}=$ desejo político do governo de efetivar a reforma agrária

$\mathrm{s}=$ investimento em violência do membro do MST

$\mathrm{v}=$ investimento em violência do membro dos fazendeiros

$\theta=$ probabilidade que o INCRA exproprie a fazenda

$\beta=$ probabilidade de sucesso da expulsão dos invasores

$\mathrm{K}=$ propensão das cortes locais a favorecer os invasores em detrimento do proprietário

$\varpi \mathrm{L}=$ valor da terra para os invasores, em caso de não-expulsão legal dos mesmos acompanhada de não-expropriação

$\delta \mathrm{L}=$ valor da terra para o fazendeiro, em caso de não-expulsão legal dos mesmos acompanhada de não-expropriação

$\gamma \mathrm{L}=$ valor da terra para o fazendeiro, em caso de expropriação

$\mathrm{L}=$ valor da terra para o fazendeiro em caso de expulsão legal (= valor da terra para os invasores em caso de expropriação legal)

$\mathrm{C}^{\mathrm{F}}(\mathrm{s})=$ custo dos fazendeiros

$\mathrm{C}^{\mathrm{F}}(\mathrm{v})=$ custo dos membros do MST

Os autores resolvem o modelo simultaneamente, encontrando as duas condições de primeira ordem abaixo: 


$$
\begin{aligned}
& (1-\beta(v, K))\left(\theta_{s} L(1-\pi)\right)=C_{s}^{F} \\
& \beta_{v}[\theta(s, P, G) L(\delta-\gamma)+L(1-\delta)]=C_{v}^{F}
\end{aligned}
$$

A solução do sistema acima gera os valores de equilíbrio para a quantidade empregada de violência por ambos os grupos. Para verificar os multiplicadores do modelo, os autores assumem algumas hipóteses sobre os parâmetros $\varpi, \delta$ e $\gamma^{32}$ e fazem uma análise de estática comparativa. Entre os diversos resultados, o principal é que a atuação governamental, por mais bem-intencionada que seja, pode ter conseqüências indesejáveis, que podem ser atribuídas à (má) formatação dos incentivos que a reforma agrária representa para os atores no meio rural.

\section{Anexo 2}

\section{Correlações Bivariadas}

\begin{tabular}{llllllll}
\hline & conf & ocup & assent00 & assent04 & mod & pob & cresc_m \\
\hline conf & 1.0000 & & & & & & \\
ocup & 0.1859 & 1.0000 & & & & & \\
assent00 & 0.0762 & 0.1611 & 1.0000 & & & & \\
assent04 & 0.3504 & 0.2225 & -0.0116 & 1.0000 & & & \\
mod & 0.0675 & 0.2263 & 0.1198 & 0.0850 & 1.0000 & & \\
pob & 0.0164 & 0.0566 & 0.0252 & 0.0313 & 0.5101 & 1.0000 & \\
cresc_m & -0.0407 & -0.1302 & -0.0990 & -0.0879 & -0.0604 & 0.0738 & 1.0000 \\
d_pt & 0.0406 & -0.0309 & 0.0082 & 0.0932 & 0.0199 & 0.0204 & -0.0226 \\
\hline
\end{tabular}

Fonte: Elaboração própria.

Anexo 3

Efeito Interação Conflito (Gráficos 1 e 2)

\begin{tabular}{llllll}
\hline Variable & Obs & Mean & Std. Dev. & Min & Max \\
\hline _probit_ie & 853 & -.0004329 & .0003856 & -.0022582 & .000039 \\
_probit_se & 853 & .0010985 & .0003665 & .0002733 & .0029204 \\
_probit_z & 853 & -.3423697 & .1880673 & -.8066572 & .0845672 \\
\hline
\end{tabular}

$\overline{{ }^{32} \text { Especificamente, } \varpi=\gamma=0 \text { e } \delta=1 .}$ 
Efeito Interação Ocupação (Gráficos 3 e 4)

\begin{tabular}{llllll}
\hline Variable & Obs & Mean & Std. Dev. & Min & Max \\
\hline _probit_ie & 853 & -.0008963 & .0018226 & -.004317 & .0078659 \\
_probit_se & 853 & .0016842 & .0022772 & $4.17 \mathrm{e}-06$ & .0098097 \\
_probit_z & 853 & -1.580049 & .9541501 & -3.059649 & 1.421989 \\
\hline
\end{tabular}

Efeito Interação Assentamento 2000 (Gráficos 5 e 6)

\begin{tabular}{llllll}
\hline Variable & Obs & Mean & Std. Dev. & Min & Max \\
\hline _probit_ie & 853 & .0008949 & .0014518 & $1.53 \mathrm{e}-09$ & .0077883 \\
_probit_se & 853 & .001191 & .0014548 & $1.33 \mathrm{e}-08$ & .0068311 \\
_probit_z & 853 & .4963444 & .2281158 & .0260754 & 1.239134 \\
\hline
\end{tabular}

Efeito Interação Assentamento 2004 (Gráficos 7 e 8)

\begin{tabular}{llllll}
\hline Variable & Obs & Mean & Std. Dev. & Min & Max \\
\hline _probit_ie & 853 & .0023867 & .0024588 & $7.04 \mathrm{e}-07$ & .0116483 \\
_probit_se & 853 & .0017112 & .0018114 & $2.98 \mathrm{e}-06$ & .0080992 \\
_probit_z & 853 & 1.346714 & .3380449 & .2361105 & 1.846941 \\
\hline
\end{tabular}

\section{Gráfico 1}

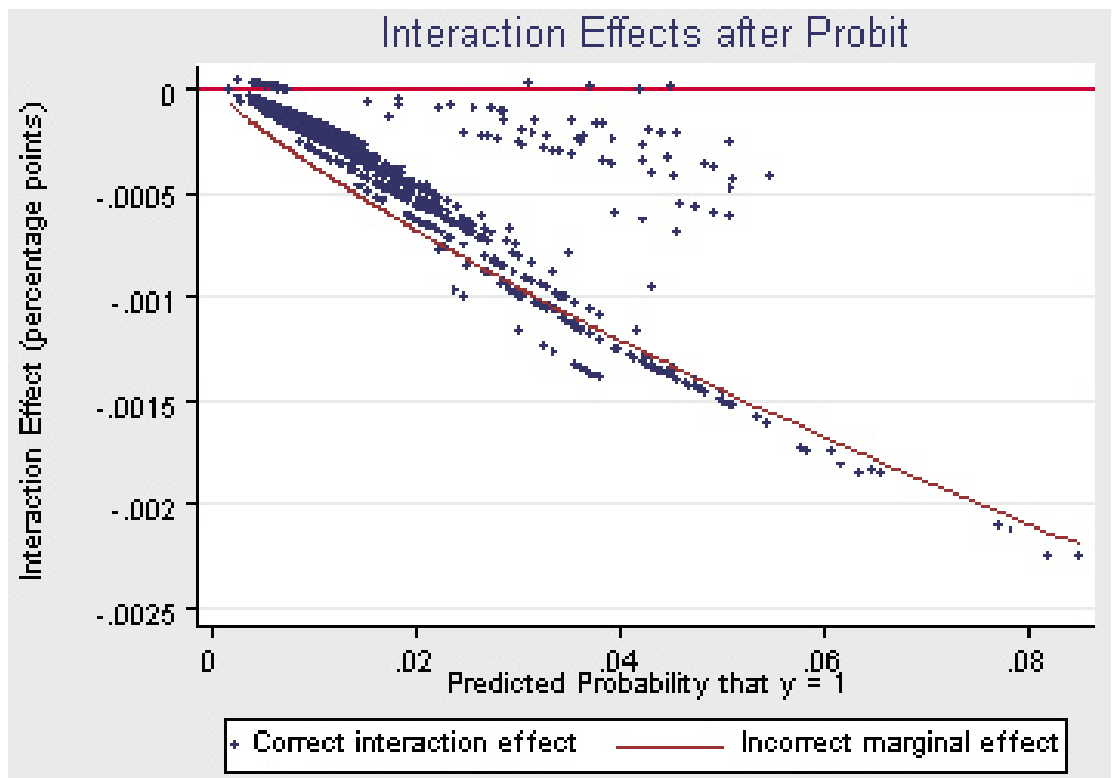


Gráfico 2

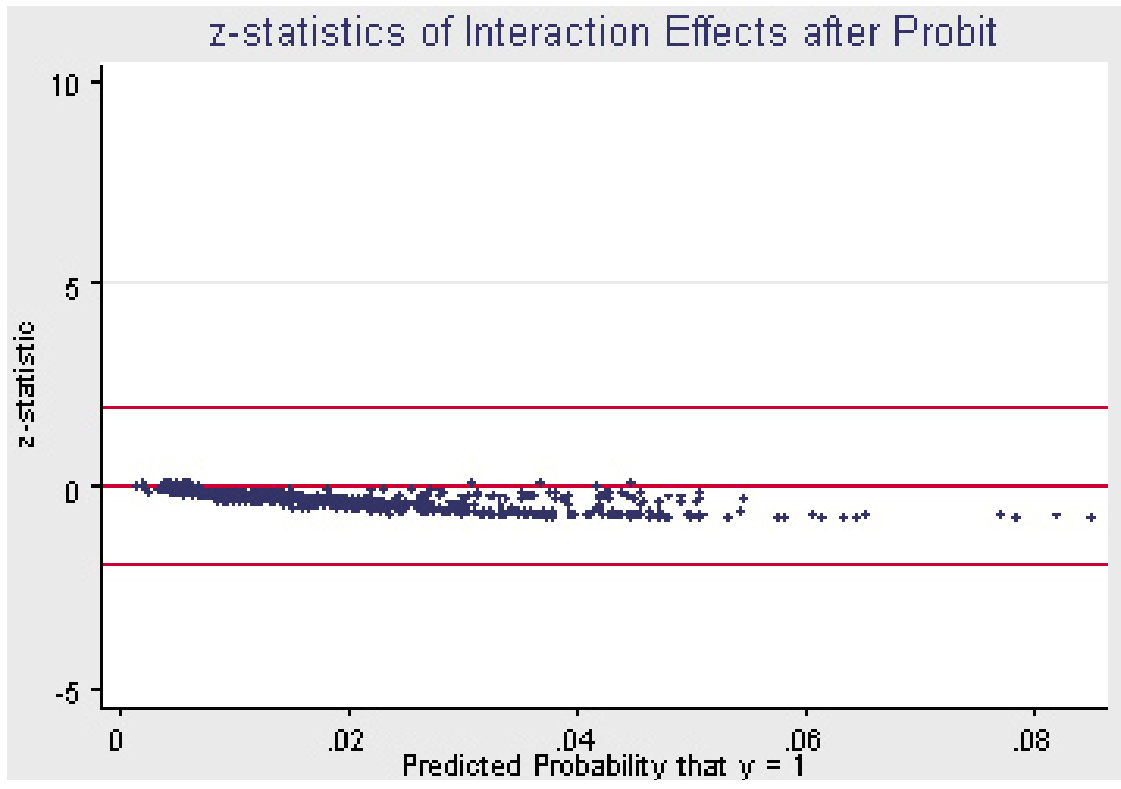

Gráfico 3

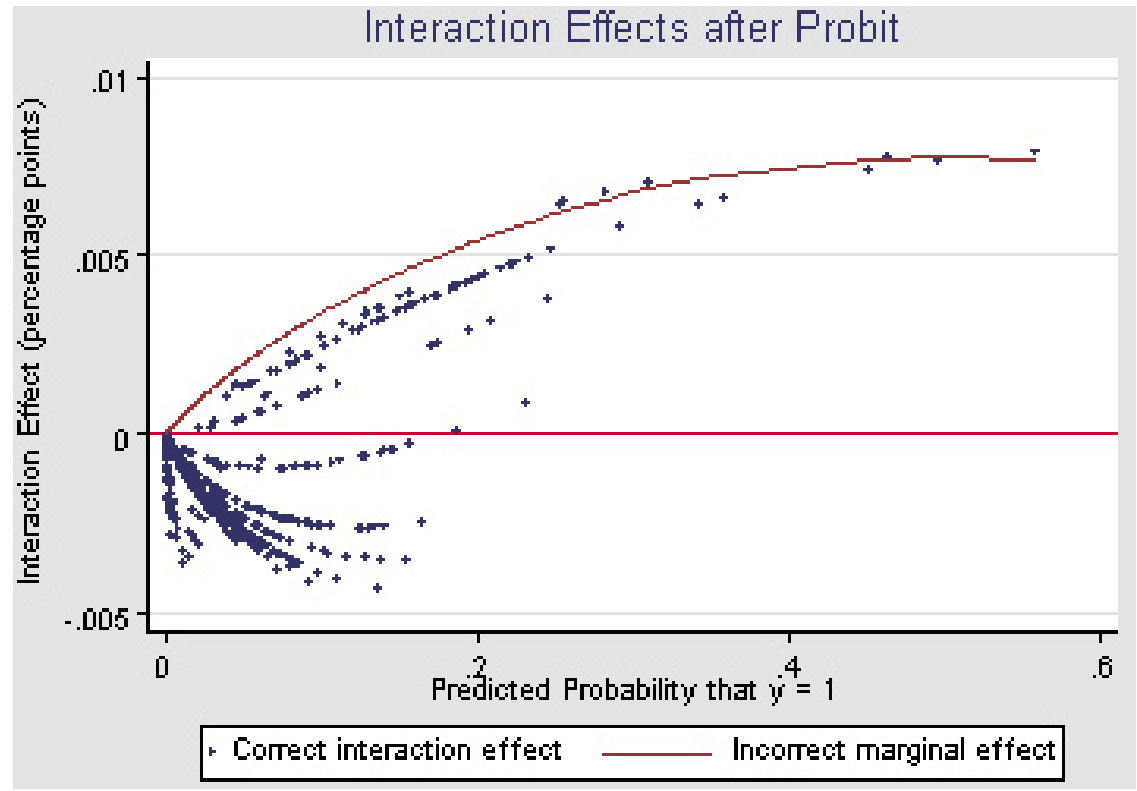


Gráfico 4

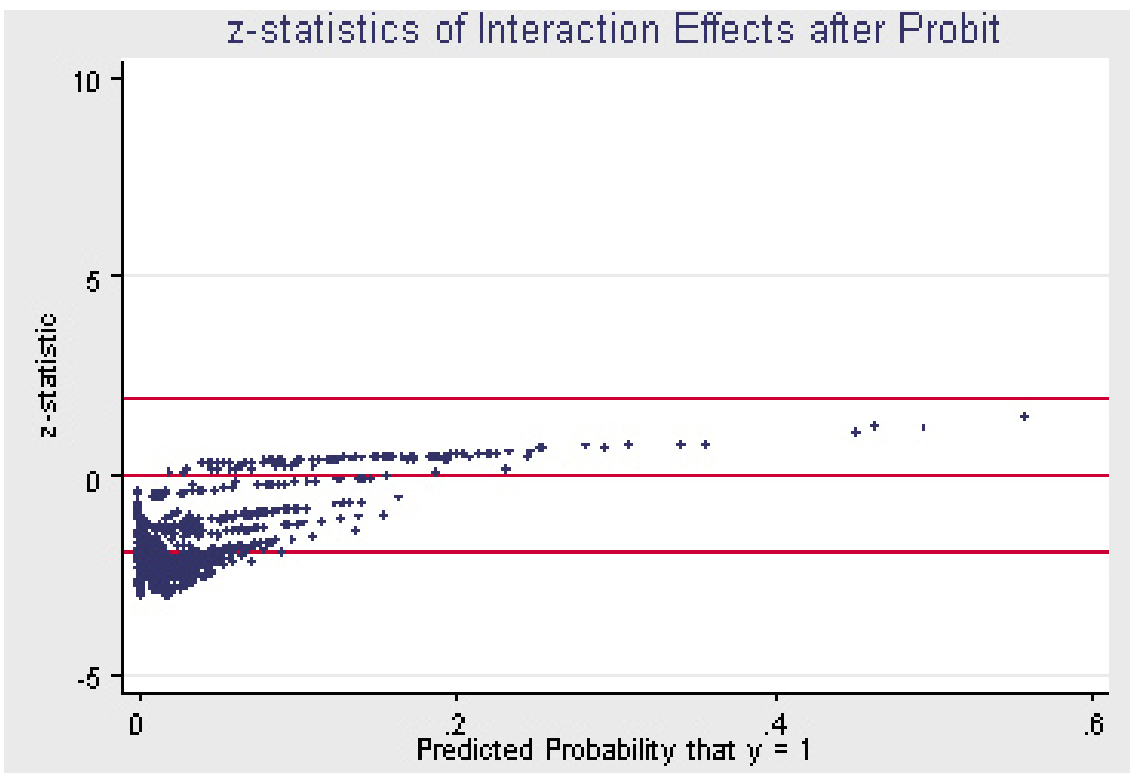

Gráfico 5

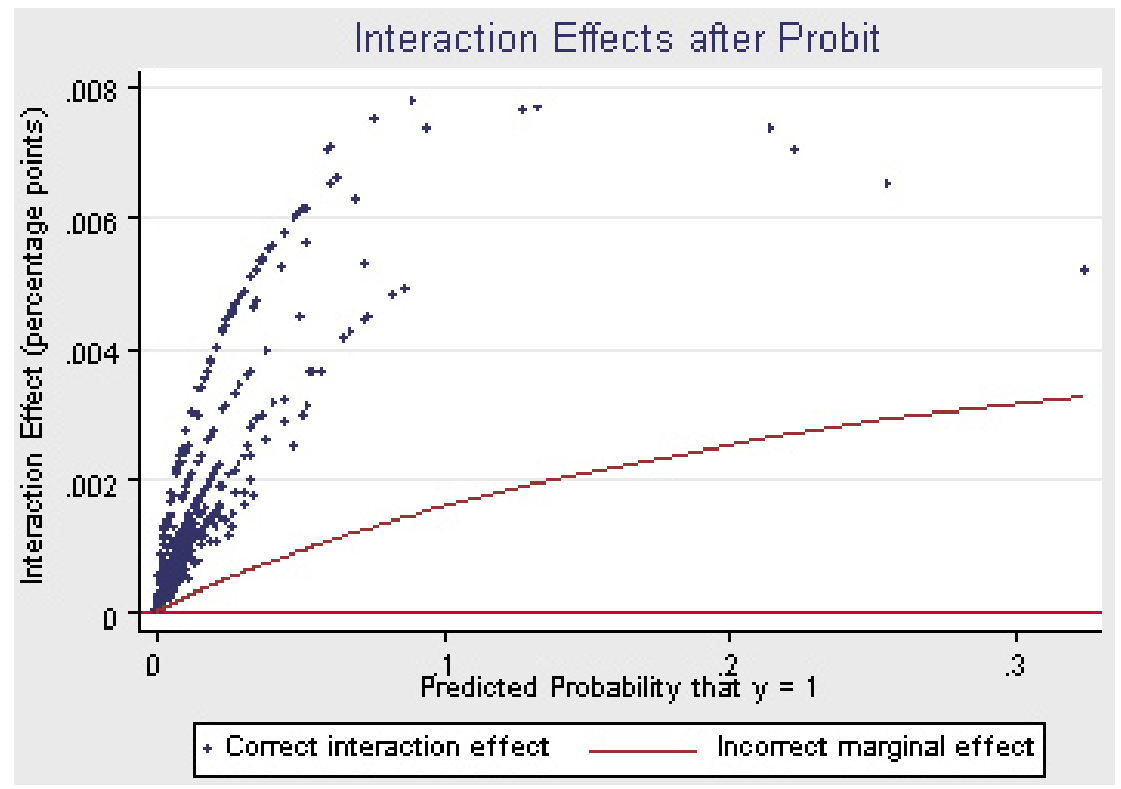


Gráfico 6

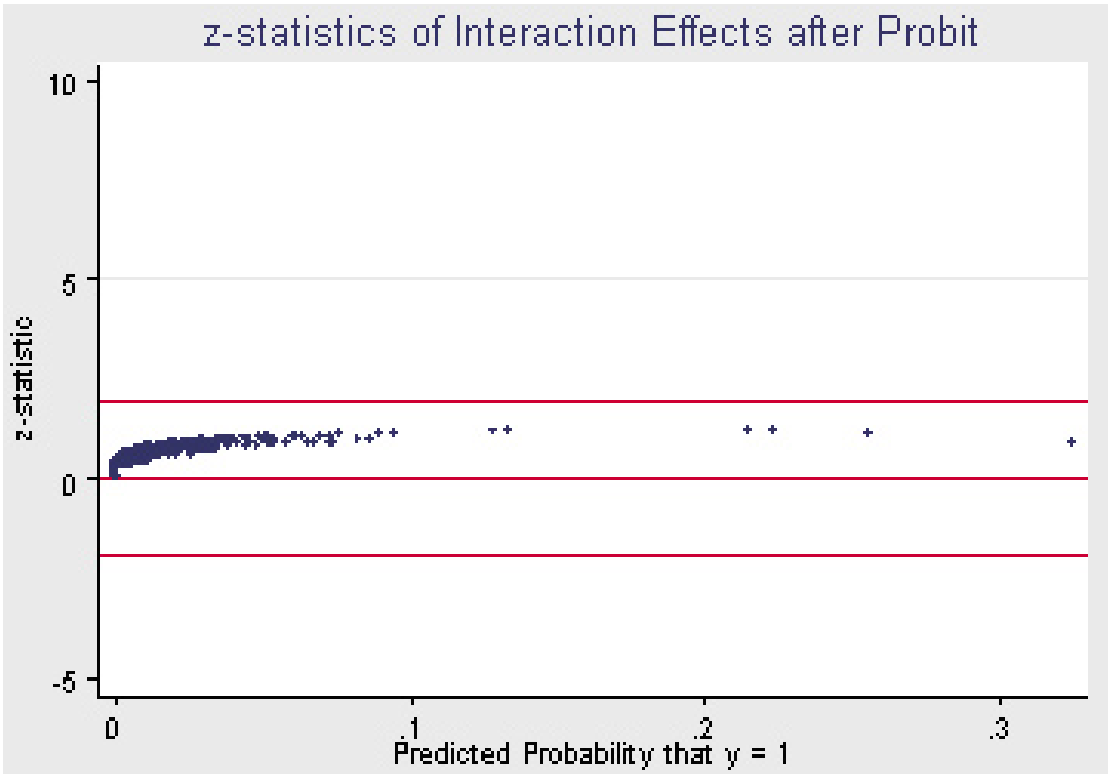

Gráfico 7

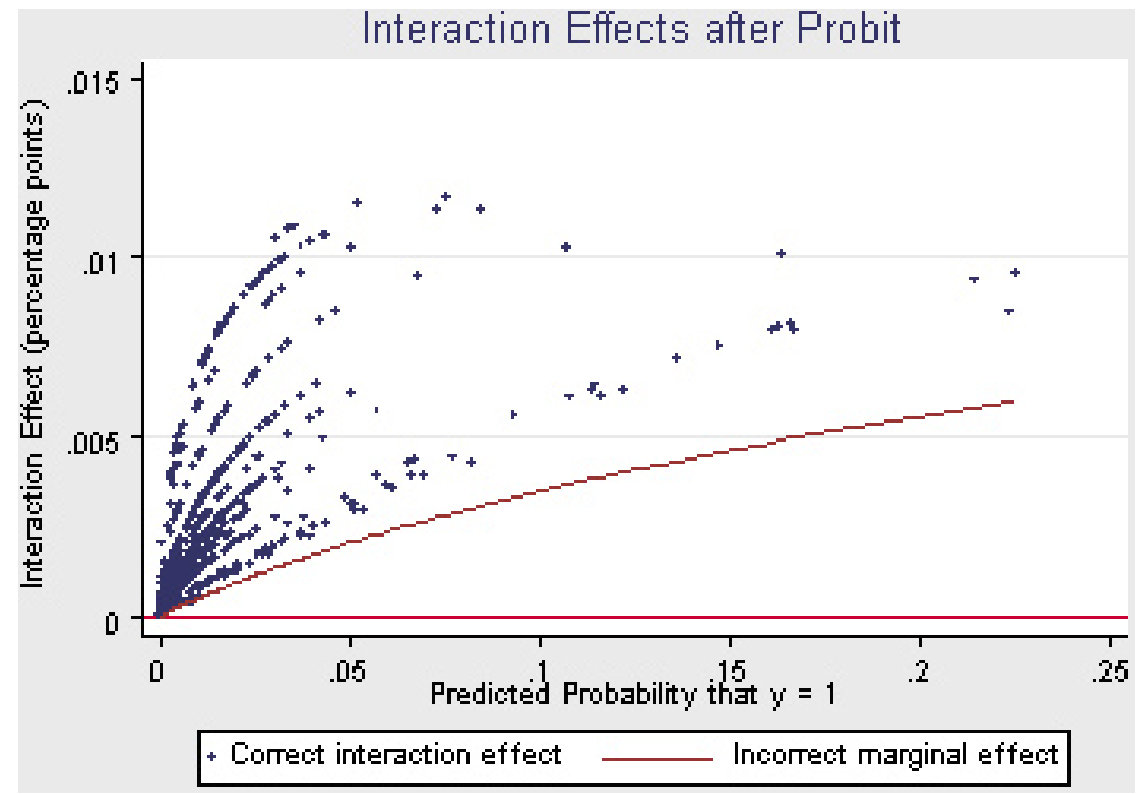


Gráfico 8

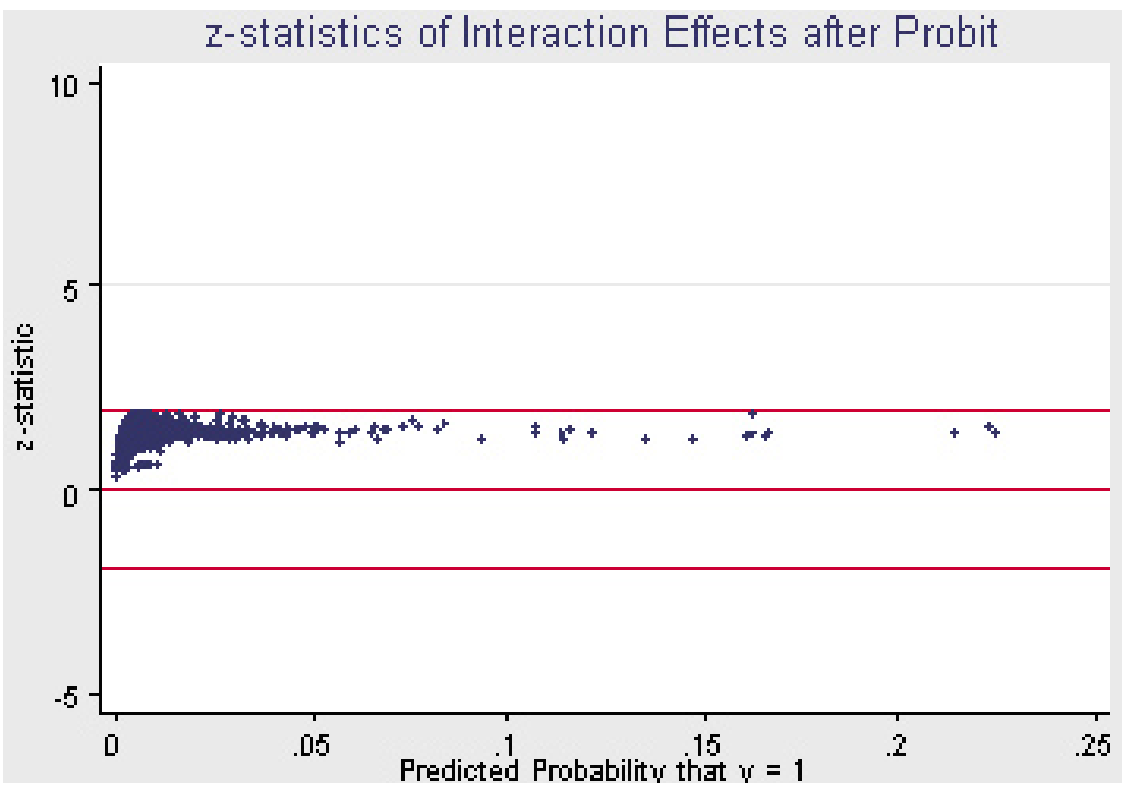

